\title{
Detection and Classification of Abnormal Respiratory Sounds on a Resource-constraint Mobile Device
}

\author{
Chinazunwa Uwaoma \\ Department of Computing \\ Faculty of Science and Technology \\ The University of the West Indies \\ Kingston, Jamaica
}

\author{
Gunjan Mansingh \\ Department of Computing \\ Faculty of Science and Technology \\ The University of the West Indies \\ Kingston, Jamaica
}

\begin{abstract}
Abnormal breath sounds like wheezes, crackles and stridor at times manifest similar morphologies and pathological features of lung airways obstruction. This may pose problems to proper diagnosis and evaluation of the underlying respiratory condition by human auscultation. In this study, the authors experimented with Time-Frequency threshold-dependent (TFTD) algorithm for detection and classification of breath sounds based on Smartphone. The TFTD algorithm computes important and distinct features of each breath sound using spectro-temporal analysis of recorded lung sounds which can enhance qualitative measurement and quantitative indexing of different respiratory sounds. Several algorithms which run exclusively on desktop computers have been developed for detecting and analyzing specific lung sounds such as wheezes. However, few attempts have been made to perform such analysis on portable devices like mobile phones due to computational complexities and high power consumption associated with the analyses. Our experimental results demonstrate that recent smartphones with improved computational capacity are able to provide comparative performance on analysis of respiratory signals. Furthermore, these phones can serve as convenient tools for measuring and detecting early signs of pulmonary disorders particularly at home and during ambulatory care services where conventional and specialized medical devices may not be accessible.
\end{abstract}

\section{General Terms}

Smartphones, Algorithms, Wheezes.

\section{Keywords}

Respiratory sounds, smartphone, auscultation, detection, abnormal, algorithm, analysis,computational capacity.

\section{INTRODUCTION}

Sounds heard during breathing can provide vital information on the condition of the respiratory organ. Adventitious respiratory sounds like wheezes, crackles and stridor usually are indicators of pulmonary disorders. Though auscultation has remained the general practice by physicians to diagnose and evaluate patients with respiratory conditions, the ability to provide vital diagnostic information through human auscultation is dependent on the physicians' experiences and interpretations [1]. Furthermore, these sounds may appear together on the same breath signal and may have similar features pointing to different respiratory diseases which makes it difficult for clinicians to identify the actual pathology of the underlying ailment. Computerized breath sound analysis on the other hand, does not only provide objective and vital information for diagnosis but also simplifies the task of medical personnel in managing their patients [2]. Modern technology in mobile computing could be used to augment traditional medical practices by automating the recognition of breathing sounds. This is realized as an embedded system in portable devices which can assist in remote monitoring by medical personnel in an $\mathrm{m}$ Health setting.

Each respiratory condition is associated with a particular breath sound and can be distinguished from other pulmonary diseases from acoustic features- tone, amplitude, pitch, duration and frequency. Automated respiratory sound analysis allows for better parameterization of these quantities and their evaluation based on their significance on the signal of interest. Recent studies [3], [4] and [5] have recorded major breakthrough in the development and improvement of computerized wheeze detection and analysis using sophisticated algorithms which involve detection of Fourier peaks and image analysis of the resulting spectrogram. Because these algorithms require enormous computational and sustainable power resources, the analyses were basically run on PCs and servers with few deliberate attempts on wearable low-power devices [6]. Besides, the approaches used did not include detection and evaluation of other abnormal sounds which may present similar morphologies alongside wheeze formation in a respiratory signal.

Building upon these observations, we took further steps in our study to develop a hybrid algorithm that can analyze different recorded lung sounds by computing and indexing their spectro-temporal features solely on intelligent mobile phones with appreciable computational and storage capacity. These phones can serve as assistive tools for measuring and detecting early signs of pulmonary disorders in scenarios where conventional and specialized medical devices may not be accessible [7]. Furthermore, identifying different patterns in the formation, duration and appearance of adventitious sounds on normal breath signal could provide additional information to physicians in the diagnosis and treatment of respiratory conditions.

\section{RELATED WORK}

Studies in recent years have focused on the use of recording devices to collect and analyze lung sounds. In these studies different signal processing methods were used to identify physiological mechanisms associated with different breath sounds and their pathological links to various pulmonary ailments [8]. Extensive research has been carried out on detecting and analyzing wheezes in respiratory signals. Timefrequency (TF) technique and wavelet packet decomposition 
(WPD) in [4] were used to analyze breath sounds which included asthma wheeze. The experimental results showed high accuracy of wheeze detection with low computational complexity. Authors in [6] focused on the analysis of computational complexity of common features extracted from Short Term Fourier Transform (STFT) using decision tree classification model. Four different algorithms and their detection accuracies were evaluated on a dataset from prerecorded respiratory sounds - wheezing in particular, with different efficiency metrics. Based on the best classification accuracy, the authors proposed a wearable wireless sensor system for automated recognition of respiratory sound patterns which is implemented on a digital signal processor (DSP) requiring low power. The system is to enable long-term monitoring of symptoms of respiratory conditions such as asthmatic wheezing. It is important to note here that these studies have concentrated mainly on the development and efficiency of different wheeze detection algorithms with little or no attention given to other abnormal respiratory sounds with similar characteristics to wheeze features, which may be present at the same time in a respiratory sound. In addition, though some of the proposed detection systems involve the use of mobile phones; both data acquisition and processing are performed outside the phone. These are the primary issues we attempt to address in this study.

\section{MATERIALS AND METHODS}

\subsection{Materials}

\subsubsection{Method of Data Acquisition}

Recorded lung sounds used for the detection and analysis of abnormal breath sounds were obtained from a repository [9] on licensed agreement. The smartphone was also used to record simulated 'wheeze' and normal breath sounds from healthy individuals to compare with the clinical recordings of pathological respiratory sounds from the database. Factors that influenced the measurement of the recorded sounds include: ambient noise, age of the subject, breathing flow, position of the patient, type of measuring device, and the location of sound capturing on the body.

\subsubsection{Development Tools}

The algorithm was implemented using Android Development Tools (ADT) running in Eclipse Environment on Windows 8.1 Computer with installed memory of $4 \mathrm{~GB}$. The resulting code was tested on an emulator - Android Virtual Device (AVD) configured on 4.4.2 platform (API 19) and later ported to Android phone running on v4.2.2 of the Operating System with 2GB of RAM. Excel 2013 provided the environment for statistical analyses.

\subsection{Methodology}

Different lung sounds from the repository were exposed to the TFTD algorithm. The signals were pre-filtered, amplified and digitalized into WAV formats with varying sampling rates and bit rates. The corresponding sampling and bit rates for each WAV file are provided in the property list. However, the sound files were down-sampled to $8000 \mathrm{~Hz}$ to lessen the processing load on the smartphone. Average signal length of the breath sounds was 10 seconds.

Figure 1 shows a block diagram of TFTD algorithm for the detection, analysis and classification of lung sounds. The algorithm is a modification of the Adaptive Respiratory Spectrum Correlation Coefficient (RSACC) [5]. In the algorithm, we used wheeze detection mechanism as a benchmark for identification of other abnormal sounds in a respiratory signal. Extensive studies haves been carried on wheeze detection and analysis in breath sounds given its clinical importance and pathological link to most common respiratory conditions like asthma, COPD, and croup.

The major task of the TFTD algorithm is to classify the recorded signals into normal respiratory sounds, baseline (ambient noise) and wheeze sounds. The algorithm also identifies the durations of the respiratory sounds and wheeze sounds and then determine the proportion of wheeze in the respiratory cycle.

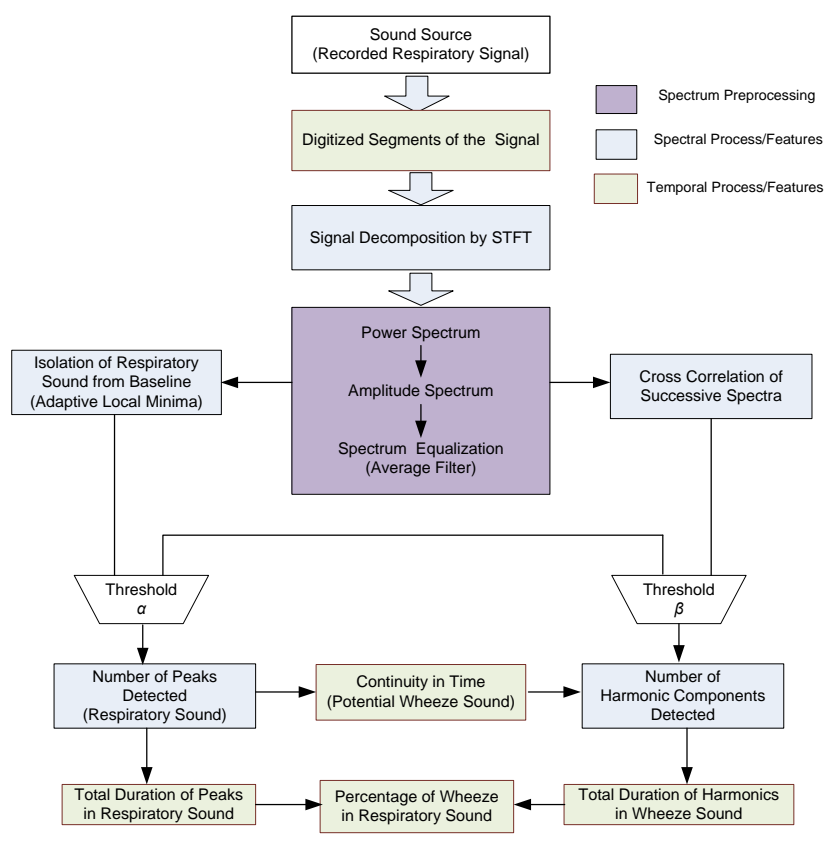

Figure 1: Process Flow of the TFTD algorithm for breath sound analysis

In implementing the algorithm, each of the recorded breath signals is segmented into frames $\mathrm{S}(\mathrm{n})$, of equal length samples $(128 \mathrm{~ms})$ with $87.5 \%$ overlap; $\mathrm{n}$ is segment number. Discrete Short Time Fourier Transform in equation (1) is performed on each of the segment to determine the time-dependence of the signal in the frequency domain. To reduce spectral leakage we used a hamming window of size $N=1024$ shifted over the signal by step $m$.

$$
P[n]=\sum_{-\infty}^{\infty} s[n] w[n-m] e^{-j 2 \pi k / N}
$$

Next, the resulting power spectrum is represented as amplitude spectrum. This is to moderate the attenuation of low magnitude frequency components that may contain potential high frequency harmonics of the signal of interest. A box filter of width three is applied to remove signal fluctuations resulting to a smoothed signal $s T(n)$. An empirically determined threshold [5] $\alpha$, is used to separate actual respiratory sounds from baseline signal by finding the local minimal value of $s T(n-2), s T(n-1)$ and $s T(n)$. The value of $\alpha$ is adaptively adjusted if $s T(n-1)$ is less than $s T(n)$, $s T(n-2)$ and the current value of $\alpha$ as shown in equation (2). The initial value of $\alpha$ is set at 0.01 which is presumed to be the maximum amplitude of ambient noise though it varies with the measuring device. 


$$
\alpha=\left\{\begin{array}{cc}
0.01, & \text { initial value } \\
s T(n-1) * 1.25, \quad s T(n-1) & <\alpha \\
& \text { and } s T(n-1)<s T(n-2) \\
& \text { and } s T(n-1)<s T(n) \\
& \text { otherwise }
\end{array}\right.
$$

The signal segment $\mathrm{S}(\mathrm{n})$ is set as respiratory sound if $s T(n)$ is greater than the adaptive threshold $\alpha$, otherwise, it is regarded as a baseline signal. To determine the presence of wheeze, cross correlation is performed on spectra $P(n)$ and $P(n-1)$. A scenario where each spectrum "exhibits continual harmonic components along the time axis" [5] such that the spectra show high resemblance, is indicative of wheeze sound. The resulting correlation coefficients are denoted by $K(n)$. Here, a threshold $\beta$, close to 1 is set on the $K(n)$ values and if three successive values of $K(n)$ that is, $K(n-2)$ to $K(n)$ exceed $\beta$ and the corresponding segment of the smoothed signal $s T(n)$ is identified as a respiratory sound, then the signal segment is classified as a wheeze sound. The respiratory sound duration and the wheeze duration are estimated and finally, wheeze occupation in the respiratory cycle is calculated as a percentage ratio of the respiratory sound.

Table 1. Comparison of different breath sounds exposed to TFTD Algorithm

\begin{tabular}{|c|c|c|c|c|c|c|c|}
\hline \multirow{2}{*}{ CAT. } & \multicolumn{3}{|c|}{$\begin{array}{l}\text { Recorded Breath } \\
\text { Sound }\end{array}$} & \multicolumn{4}{|c|}{ After Processing } \\
\hline & Label & DP & $\begin{array}{c}\text { Len } \\
(s)\end{array}$ & Segs. & RSS & WSS & $\begin{array}{l}\text { WP } \\
(\%)\end{array}$ \\
\hline \multirow{2}{*}{ 胥 } & N_01 & $\mathrm{n} / \mathrm{a}$ & 10 & 624 & 405 & 69 & 17.0 \\
\hline & N_02 & $\mathrm{n} / \mathrm{a}$ & 10 & 611 & 405 & 76 & 18.9 \\
\hline \multirow{2}{*}{ 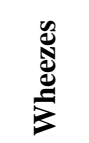 } & W_01 & VB & 10 & 618 & 404 & 95 & 23.5 \\
\hline & W_02 & AS & 20 & 1249 & 407 & 132 & 32.4 \\
\hline \multirow{2}{*}{ 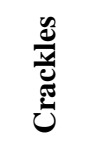 } & C_01 & $\mathrm{CF}$ & 10 & 624 & 386 & 53 & 13.7 \\
\hline & C_02 & $\mathrm{PN}$ & 10 & 624 & 394 & 58 & 14.7 \\
\hline \multirow{2}{*}{ 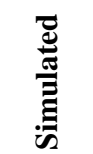 } & S_01 & $\mathrm{n} / \mathrm{a}$ & 7 & 414 & 0 & 0 & 0 \\
\hline & S_02 & $\mathrm{n} / \mathrm{a}$ & 4 & 217 & 0 & 0 & 0 \\
\hline
\end{tabular}
each respiratory sound, Len: Signal length in seconds, DP: Diagnosed Pathology, Segs: Total number of segments in each signal RSS: Respiratory Sound Segments, WSS: Wheeze Sound Segments, WP: Percentage wheeze proportion in respiratory sound, $\boldsymbol{A S}$ : Asthma, VB: Viral Bronchiolitis, CF: Cystic Fibrosis, PN: Pneumonia.

\section{RESULTS AND DISCUSSION}

One of the specific objectives of our study is to develop algorithm that can distinguish feature patterns of abnormal sounds which may manifest similar morphologies in a respiratory signal. We accomplished this by extracting and comparing dominant frequency ranges of different breath

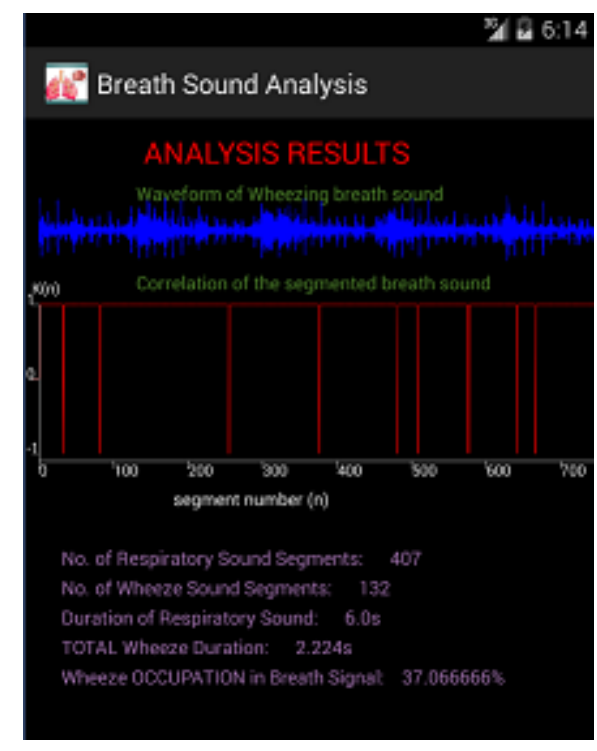

(a)

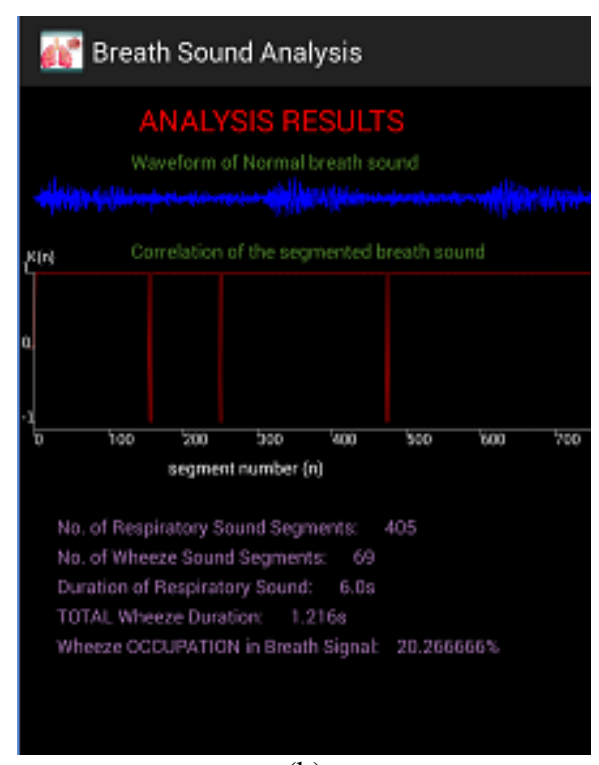

(b)

Figure 2: Plots of waveform and correlation of detected peaks on Smartphone. (a)wheezes (b) normal

sounds, the cross correlation coefficients of the energy spectra and continuity in time. Three important lung sounds often observed in health and disease were selected for the analysis. The waveform, spectrogram and cross correlation for respiratory sounds selected from each of the categories are shown in Figures 2 and 4.

We adopted two analysis approaches namely, qualitative measure and quantitative description. The QUALITATIVE MEASURE basically involves visualization of the sound waveform, spectrogram and cross correlation plot (resulting from the TFTD algorithm) and listening to audio data. Criteria used for evaluation include clarity of sound which requires elimination of ambient noise and false peaks in the timefrequency plane; and preservation of sound morphology which involves elimination of distortion in terms of frequency 
bandwith and time duration in the time-frequency domain. The evaluation procedures were carried out by expert physicians as documented in [9]. Parameters used for QUANTITATIVE DESCRIPTIONS include: sound duration, maximum number of harmonics, and peaks in dominant frequency range.

For abnormal sound detection, we focused mainly on wheezes and crackles which are the more studied adventitious sounds in pulmonary medicine. We describe briefly the characteristics of these two sounds as follows:

Wheezes: Continuous, musical sounds heard mostly during expiration with duration between $100 \mathrm{~ms}$ and $250 \mathrm{~ms}$. The fundamental frequency ranges between $100 \mathrm{~Hz}$ and $1000 \mathrm{~Hz}$. Wheezes have distinct frequencies with dominant frequencies with dominant frequencies around $400 \mathrm{~Hz}$ (see Figure 3). The wave form is usually sinusoidal. Stripe patterns of harmonic components are usually observed on the STFT graph of wheezing sound which distinguishes it from other normal breath sounds as shown in Figure 4. Principal pathologies include asthma, croup, COPD and emotional laryngeal stenosis [8].

Crackles: Crackles on the other hand, are non-stationary or discontinuous short explosive sounds observed in patients with pneumonia, pulmonary fibrosis and COPD. The duration is less than $10 \mathrm{~ms}$ and often inspiratory. Unlike wheezes, crackles do not have distinct frequencies but broad distributed frequencies on the spectrogram ranging between $600 \mathrm{~Hz}$ and $2000 \mathrm{~Hz}$ (see Figures 3 and 4). The signal has a short but wide deflected waveform.

The analysis shown in Table 1 was based on number of consecutive harmonics in the signal spectra and their total duration. As in the previous researches [1], [3], [4] and [5] on wheeze detection, the harmonic pattern of the spectrum is one of the important features used to identify the appearance of wheeze in respiratory signal. And since the algorithm was built around wheeze detection, the threshold for harmonic counts were based on the maximum number of co-existing peaks (harmonic components) in a wheeze [3] which should not be greater than 3 with the peak of the fundamental frequency making a total of 4 . For a segment of the respiratory signal to be classified as wheeze, the duration must at least be greater than $150 \mathrm{~ms}$.

About 8 different breath sounds were tested on the TFTD Algorithm with equal number of sounds representing each category. Data from Table 1 shows that the number of sound segments classified as Respiratory Sound (RSS) were within a close range of 400 irrespective of the signal length. This goes to show the insusceptibility of the algorithm to ambient sounds. This robustness was typified in the category of simulated breath sound showing 0 RSS in both cases. However, we observed strong correlation of respiratory signals between wheeze sounds and other respiratory sounds; hence, the reason for false detection of wheezes in the categories of normal breath sounds and crackles. The false alarm rate in the entire process was $10.6 \%$. Notwithstanding, wheezing sound has the highest number of harmonic counts = 4 as revealed in the histogram plot in Figure 5. To reduce the rate of erroneous detection, we incorporated into the algorithm another spectral feature which is dominant frequency range pegging the threshold as it applies to wheezes and crackles.

An important factor that influenced the outcome of the algorithm test was the position of the recording device mainly on the trachea and the chest. The measurement position of each respiratory sound was clearly stated in the database [9] from where we obtained the sound samples we used for the experiment. Because wheezes usually emanate from the lungs, most of the recordings were performed on the chest and it has been noted that the chest wall functions as low pass filter that attenuates high frequencies, and this implies that high pitched wheezes could be lost in the recordings. On the other hand breath sounds recorded on the trachea show higher frequency components with more pronounced peaks compared to recording performed on the chest wall.

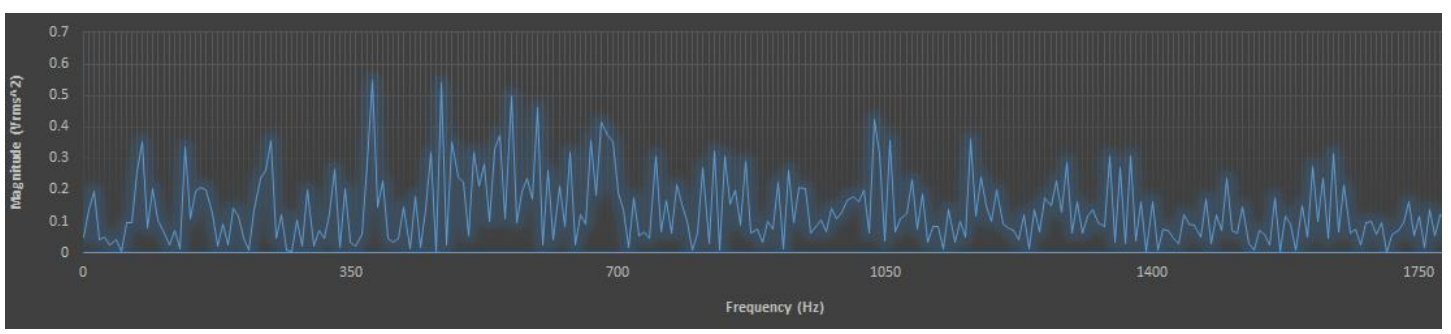

(a)

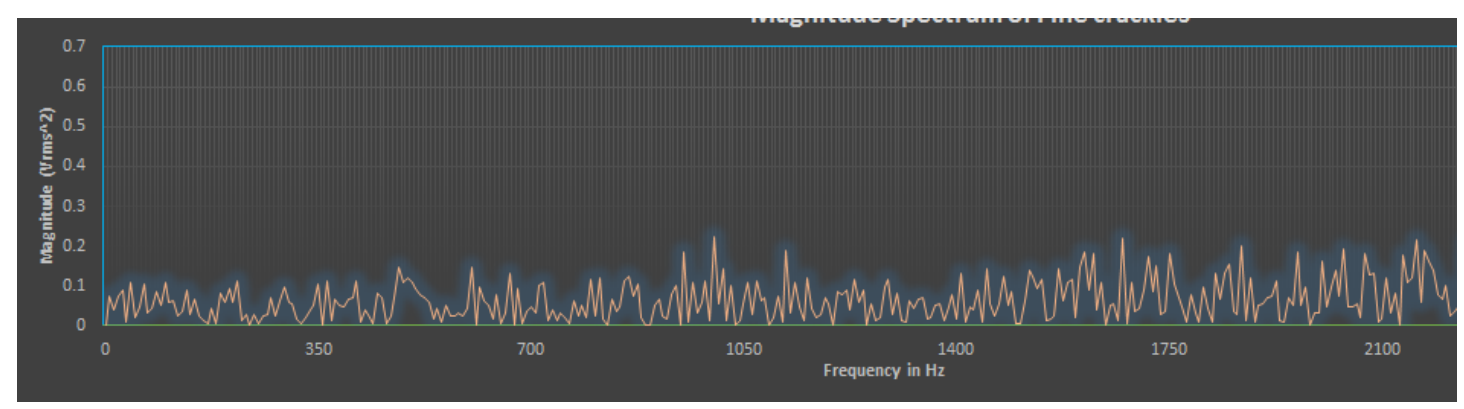

(b)

Figure 3: Expanded magnitude plot showing frequency distribution in (a) wheezes, (b) crackles 


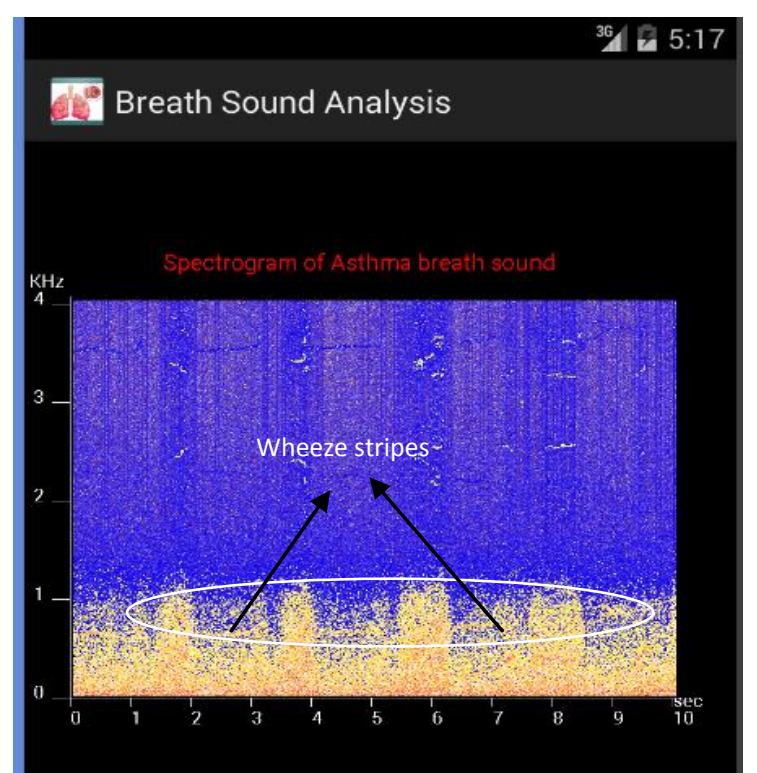

(a)

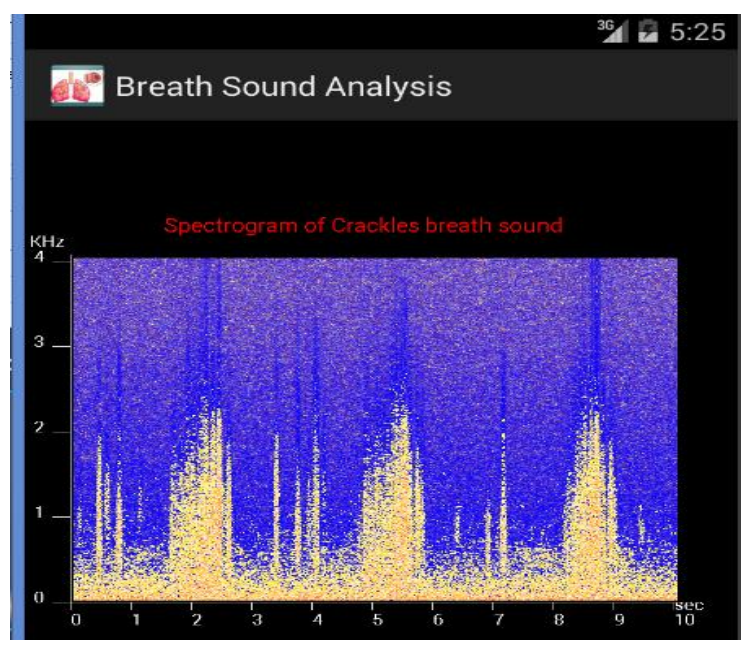

(b)

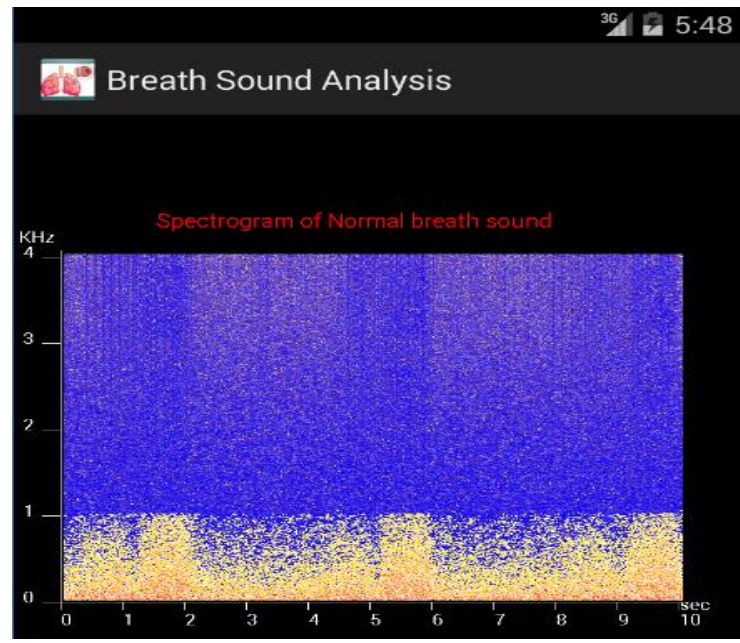

(c)

Figure 4: Spectrogram Plots (a) wheeze (b) crackles (c) normal

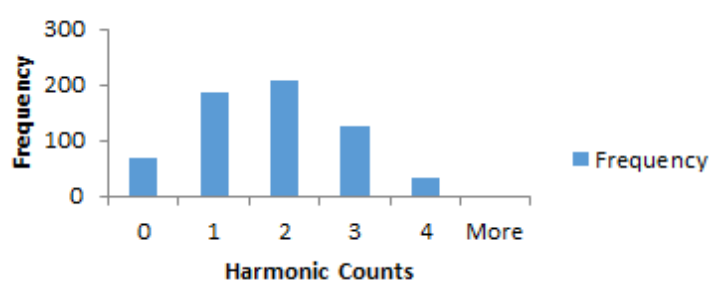

(a)

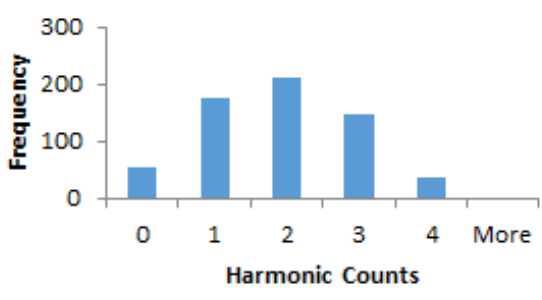

Frequency

(b)

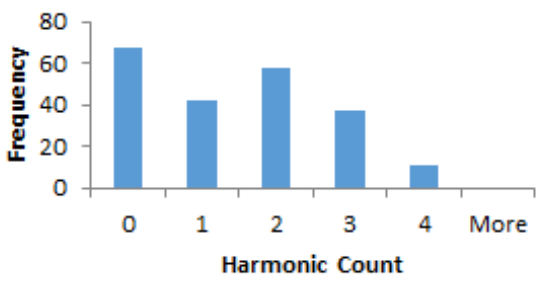

Frequency

(c)

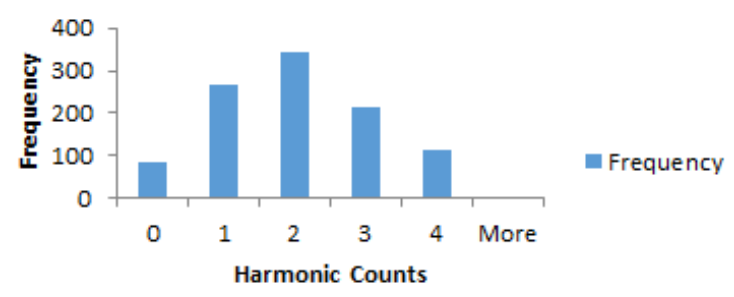

(d)

Figure 5: Histogram Plots of Harmonic Counts (a) crackles (b) normal (c) simulated (d) wheezes

Both the detection and classification of the breath sounds were performed on the TFTD algorithm. However, there is need to search for optimal parameters using classification models such as multilayer perceptron (MLP) neural network and k-nearest neighbor (k-NN) through machine learning; which will help provide further information on the statistical metrics - sensitivity, specificity and accuracy of the detection and classification processes. We hope to use these techniques in our future study.

\section{CONCLUSION}

In this article, we have provided a proof-of-concept that intelligent mobile phones are capable of recording and analyzing breath sounds solely without any recourse to backend systems or other networked devices. In the experiment however, we noted false wheeze detection due to existence of peaks in other breath sounds which are highly similar to peaks representing wheezes. The algorithm can be improved by extracting more spectral features but not without additional computation overhead. The study is an ongoing 
research and we intend to implement the findings in a project that has to do with creating a personal healthcare system for monitoring and detection of early symptoms of asthma, using smartphone as platform for data acquisition, analysis and presentation. And with the increasing acceptance of m-Health in modern medicine, we believe that the systematic approach we presented in this work can assist health professionals in recognizing the underlying pathology of the abnormal sounds often heard in patients with respiratory conditions.

\section{ACKNOWLEDGMENTS}

The authors would like to thank LIME Jamaica for providing the mobile devices used for the preliminary testing of the algorithm. The recorded lung sounds were exclusively obtained with authorization from Medi-Wave Inc. Winnipeg, Canada.

\section{REFERENCES}

[1] Shaharum, S. Sundaraj, K., and Palaniappan, R. 2012. "A survey on automated wheeze detection systems for asthmatic patients." Bosnian Journal of Basic Med. Sci. vol. 12 , no. 4 , pp. $249-255$

[2] Lin, B., and Yen, T. 2014. "An FPGA-Based Rapid Wheezing Detection System." International journal of environmental research and public health, vol. 11, no. 2, pp. 1573-1593.

[3] Taplidou, S., and Hadjileontiadis, L.J. 2007. "Wheeze detection based on time-frequency analysis of breath sounds," Computers in biology and medicine, vol.37, no. 8, pp.1073-1083.

[4] Emrani, S., and Krim, H. 2013. "Wheeze Detection and Location using Spectro-temporal Analysis of Lung Sounds." In Proceedings of 29th Southern Biomedical Engineering Conference (SBEC), IEEE, 2013, pp. 3738.

[5] Yu, C., Tsai, T., Huang, S., and Lin, C. 2013. "Soft Stethoscope for Detecting Asthma Wheeze in Young Children." Sensors, vol. 13, no. 6, pp. 7399-7413.

[6] Oletic, D., Arsenali, B., and Bilas, V. 2014. "LowPower Wearable Respiratory Sound Sensing." Sensors, vol. 14 no. 4, pp. 6535-6566.

[7] Uwaoma, C., and Mansingh, G. 2014. "Using Embedded Sensors in Smartphones to Monitor and Detect Early Symptoms of Exercise-induced Asthma." In Proceedings of 3rd Intl. Conf. on Sensor Networks, SENSORNETS 2014, pp. 145-150.

[8] Reichert, S., Gass, R., Brandt, C., and Andrè, E. 2008. "Analysis of respiratory sounds: state of the art." Clinical medicine Circulatory, respiratory and pulmonary medicine, vol.2, pp. $45-58$.

[9] R.A.L.E Lung Sounds. (n.d) Retrieved from: http://www.rale.ca. 